\title{
Antigen Test Positivity After COVID-19 Isolation - Yukon-Kuskokwim Delta Region, Alaska, January-February 2022
}

Brian Lefferts, $\mathrm{MPH}^{1}$; Ian Blake, MS²; Dana Bruden, MS²; Melissa B. Hagen, MD ${ }^{3,4}$; Ellen Hodges, MD ${ }^{1}$; Hannah L. Kirking ${ }^{3,4}$; Elizabeth Bates, MD ${ }^{1}$; Amanda Hoeldt ${ }^{1}$; Brenda Lamont ${ }^{1}$; Sharon Saydah, PhD ${ }^{3,4}$; Adam MacNeil, PhD ${ }^{3,4}$; Michael G. Bruce, MD ${ }^{2}$; Ian D. Plumb, MBBS 3 ,4

Isolation is recommended during acute infection with SARS-CoV-2, the virus that causes COVID-19, but the duration of infectiousness varies among individual persons. Rapid antigen test results have been correlated with detection of viable virus (1-3) and might inform isolation guidance, but data are limited for the recently emerged SARS-CoV-2 B.1.1.529 (Omicron) variant. On January 5, 2022, the YukonKuskokwim Health Corporation (YKHC) recommended that persons with SARS-CoV-2 infection isolate for 10 days after symptom onset (or, for asymptomatic persons, 10 days after a positive nucleic acid amplification or antigen test result). However, isolation could end after 5-9 days if symptoms were resolving or absent, fever was absent for $\geq 24$ hours without fever-reducing medications, and an Abbott BinaxNOW COVID-19 Ag (BinaxNOW) rapid antigen test result was negative. Antigen test results and associated individual characteristics were analyzed among 3,502 infections reported to YKHC during January 1-February 9, 2022. After 5-9 days, 396 of 729 persons evaluated $(54.3 \%)$ had a positive antigen test result, with a declining percentage positive over time. In a multivariable model, a positive antigen test result was more likely after 5 days compared with 9 days (adjusted odds ratio $[\mathrm{aOR}]=6.39)$ or after symptomatic infection $(\mathrm{aOR}=9.63)$, and less likely after previous infection $(\mathrm{aOR}=0.30)$, receipt of a primary COVID-19 vaccination series $(\mathrm{aOR}=0.60)$, or after both previous infection and receipt of a primary COVID-19 vaccination series $(\mathrm{aOR}=0.17)$. Antigen tests might be a useful tool to guide recommendations for isolation after SARS-CoV-2 infection. During the 10 days after infection, persons might be infectious to others and are recommended to wear a well-fitting mask when around others, even if ending isolation after 5 days.

YKHC provides health care and public health services to approximately 27,000 persons in an area of southwest Alaska that includes 50 remote communities; high rates of COVID-19 have been reported in this region.* On January 5,2022 , after rapidly increasing incidence of SARS-CoV-2 infections associated with introduction of the Omicron variant, ${ }^{\dagger}$ YKHC recommended an isolation policy incorporating

\footnotetext{
* https://www.ykhc.org/covid-19/situation-reports (Accessed February 12, 2022).

${ }^{\dagger}$ https://akvariants.github.io (Accessed February 7, 2022).
}

the use of SARS-CoV-2 antigen tests. ${ }^{\S}$ All persons with a positive SARS-CoV-2 antigen or nucleic acid amplification test (NAAT) result were eligible to receive a follow-up BinaxNOW SARS-CoV-2 antigen test 5-9 days after symptom onset (or, in asymptomatic persons, after the first positive test result) if the person reported no symptoms or resolving symptoms, and no fever for $\geq 24$ hours without fever-reducing medications. Persons were recommended to isolate for 10 days but could end isolation after 5-9 days if the follow-up antigen test was negative; all persons were advised to wear a well-fitting mask around others and to avoid close contact with persons at elevated risk for severe COVID-19, until the end of the 10-day period.

Persons with a positive SARS-CoV-2 NAAT or antigen test result were interviewed by a public health or clinic staff member after notification to YKHC, and at the time of a follow-up antigen test, if performed after the initial interview. Information collected included age, sex, whether the person was of the American Indian or Alaska Native race, and whether any symptoms were reported during follow-up. Persons were considered vaccinated if $\geq 14$ days had elapsed since completion of a primary COVID-19 vaccination series and were considered to have received a booster dose if $\geq 7$ days had elapsed after receipt of their booster dose. Previous infection was defined as a previous positive NAAT or antigen test result $>90$ days before the current infection episode; vaccination and previous infection status were assessed from electronic health records. Follow-up antigen testing was performed by YKHC staff members at a local health facility using the BinaxNOW antigen test'; results of the first follow-up antigen test were recorded in the electronic health record.

Positive results of the first follow-up antigen test were evaluated by demographic characteristics, symptom status, previous infection, vaccination status, and number of days since symptom onset or a positive test result. Multivariable logistic regression models were used to identify factors independently associated with a positive follow-up antigen test result, adjusted for age group, previous infection, vaccination status, presence of symptoms, and number of days since symptom onset or the initial positive test result. Statistical analyses were conducted

\footnotetext{
\$https://www.ykhc.org/wp-content/uploads/2022/01/010522-YKHCGuidance-for-Vaccinated-and-Unvaccinated-Individuals-1.pdf https://www.fda.gov/media/141570/download
} 
using SAS (version 9.4; SAS Institute), using a two-sided significance threshold of $\mathrm{p}<0.05$; univariate comparisons were made using the chi-square test. This activity was reviewed by $\mathrm{CDC}$ and was conducted consistent with applicable federal law and CDC policy.**

During January 1-February 9, 2022, a total of 3,502 persons with SARS-CoV-2 infection were reported to YKHC, including 3,266 $(93.3 \%)$ in whom symptom onset or the initial positive test result had occurred 5-9 days earlier. Among these persons, 729 (22.3\%) received a follow-up BinaxNOW antigen test 5-9 days after symptom onset or, among asymptomatic persons, after the first positive test result (Supplementary Table, https://stacks.cdc.gov/view/cdc/114423). The median age was 30 years (IQR $=17-45$ years), 380 (52.1\%) were female, and $666(91.4 \%)$ were of the American Indian or Alaska Native race. By the day of the initial positive test, 541 (74.2\%) had completed a primary COVID-19 vaccination series $\geq 14$ days earlier, including 215 (39.7\%) who had also received a booster dose; 21 (2.9\%) persons were partially vaccinated, and 167 (22.9\%) were unvaccinated. Previous infection was documented in 145 (19.9\%) persons, including 108 who had also completed a primary COVID-19 vaccination series; among persons with a previous infection, a median of 418 days (IQR = 343-439 days) had elapsed between the earlier infection and the current episode. Symptoms were reported by 564 (77.4\%) persons. Compared with persons with SARS-CoV-2 infection without follow-up antigen testing, those with follow-up antigen testing were more likely to be older (median age $=30$ years versus 22 years, $\mathrm{p}<0.001)$ and to have received a primary COVID-19 vaccine series $(74.2 \%$ versus $59.9 \%$; $\mathrm{p}<0.001)$, although other characteristics were similar.

Overall, 396 (54.3\%) tested persons had a positive BinaxNOW antigen test 5-9 days after symptom onset or after an initial positive test (Table 1). A positive antigen test was more likely after a symptomatic infection (361 of 564, $64.0 \%$ ) than after an asymptomatic infection (35 of 165 , $21.2 \%)(p<0.001)$. The proportion of positive antigen test results declined with the number of days since an initial positive test $(\mathrm{p}<0.001)$, and, among persons with symptomatic infections, since symptom onset $(\mathrm{p}<0.001)$ (Figure). A positive test result was more likely if there was no history of previous infection (346 of 584, 59.2\%) than if there was a documented previous infection (50 of $145,34.5 \%)(\mathrm{p}<0.001)$. Among 541 persons who received a primary vaccination series, 285 $(52.7 \%)$ had a positive antigen test result, including 127 of $215(59.1 \%)$ persons who had received a booster dose and 158 of $326(48.5 \%)$ who had not received a booster dose.

\footnotetext{
** 45 C.F.R. part 46, 21 C.F.R. part 56; 42 U.S.C. Sect. 241 (d); 5 U.S.C. Sect. 552a; 44 U.S.C. Sect 3501 et seq.
}

Among 167 unvaccinated persons, 98 (58.7\%) had a positive antigen test result. In multivariable models, a positive antigen test result was more likely after 5 days than after 9 days $(\mathrm{aOR}=6.39 ; 95 \% \mathrm{CI}=3.39-12.03)$, symptomatic infection $(\mathrm{aOR}=9.63 ; 95 \% \mathrm{CI}=6.03-15.37)$, and less likely after previous infection $(\mathrm{aOR}=0.30 ; 95 \% \mathrm{CI}=0.19-0.46)$, receipt of a primary COVID-19 vaccination series $(\mathrm{aOR}=0.60$; $95 \% \mathrm{CI}=0.39-0.93)$ or after both previous infection and receipt of a primary COVID-19 vaccination series $(\mathrm{aOR}=0.17 ; 95 \% \mathrm{CI}=0.09-0.33)($ Table 2$)$.

\section{Discussion}

In this study conducted after SARS-CoV-2 infection during emergence of the Omicron variant, the majority of persons with follow-up testing had a positive antigen test result 5-9 days after symptom onset, or, among asymptomatic persons, after the initial positive diagnostic test. The proportion of positive test results declined with time since infection and was lower after asymptomatic than symptomatic infections. The proportion of positive follow-up antigen test results was also lower after previous SARS-CoV-2 infection or vaccination and was lowest among vaccinated persons with a previous infection. However, the percentage of positive test results after SARS-CoV-2 infection among those who had received a booster dose was similar to that among unvaccinated persons; the reasons for this finding are unclear and might reflect differences in testing practices or other individual characteristics. Overall, these findings are consistent with other analyses of positive test results by time since infection, including a recent study in which $43 \%$ percent of health care workers with SARS-CoV-2 infection were found to have received a positive antigen test result after 5-10 days. ${ }^{\dagger \dagger}$

Persons are estimated to be most infectious approximately 4 days after SARS-CoV-2 infection (4), and SARS-CoV-2 virus generally can be cultured up to 10 days after symptom onset (3). Rapid antigen test results have previously been shown to correlate with real-time reverse transcription-polymerase chain reaction cycle threshold values (5), and with detection of viable virus, for several days after infection $(1,3,6)$. Among persons in this study with symptomatic infection, $64 \%$ received a positive antigen test result during the 5-9 days after symptom onset. However, a positive antigen test result does not necessarily mean that a person is infectious; similarly, a negative test result does not necessarily mean that a person is not infectious. Nonetheless, a positive or negative antigen test might be a useful proxy for the risk for being infectious. Therefore, lower prevalence of positive test results over time and after asymptomatic infections might reflect lower infectiousness. However,

\footnotetext{
$\overline{\dagger \dagger}$ https://www.medrxiv.org/content/10.1101/2022.02.01.22269931v1.full.pdf
} 
TABLE 1. Characteristics associated with a positive SARS-CoV-2 antigen test result 5-9 days after symptom onset or after a positive initial test result for SARS-CoV-2 - Yukon-Kuskokwim Delta region, Alaska, January-February 2022

\begin{tabular}{|c|c|c|c|}
\hline \multirow[b]{2}{*}{ Characteristic } & \multicolumn{3}{|c|}{ Antigen test result* positive, no./total no. (\%) } \\
\hline & $\begin{array}{l}\text { All infections }{ }^{\dagger} \\
\quad(N=729)\end{array}$ & $\begin{array}{l}\text { Symptomatic infections }{ }^{\dagger} \\
\qquad(n=564)\end{array}$ & $\begin{array}{l}\text { Asymptomatic infections }{ }^{\dagger} \\
(n=165)\end{array}$ \\
\hline \multicolumn{4}{|l|}{ Symptomatic $^{\dagger}$} \\
\hline No & $35 / 165(21.2)$ & - & $35 / 165(21.2)$ \\
\hline Yes & $361 / 564(64.0)$ & $361 / 564(64.0)$ & - \\
\hline \multicolumn{4}{|l|}{ Age group, yrs } \\
\hline $0-17$ & $93 / 186(50.0)$ & $83 / 131(63.4)$ & $10 / 55(18.2)$ \\
\hline $18-49$ & $216 / 400(54.0)$ & $196 / 315(62.2)$ & $20 / 85(23.5)$ \\
\hline$\geq 50$ & $87 / 143(60.8)$ & $82 / 118(69.5)$ & $5 / 25(20.0)$ \\
\hline \multicolumn{4}{|l|}{ Sex } \\
\hline Male & $180 / 349(51.6)$ & $159 / 253(62.8)$ & $21 / 96(21.9)$ \\
\hline Female & $216 / 380(56.8)$ & $202 / 311(65.0)$ & $14 / 69(20.3)$ \\
\hline \multicolumn{4}{|l|}{ Race and ethnicity } \\
\hline American Indian or Alaska Native & $361 / 666(54.2)$ & $329 / 513(64.1)$ & $32 / 153(20.9)$ \\
\hline Other & $35 / 63(55.6)$ & $32 / 51(62.7)$ & $3 / 12(25.0)$ \\
\hline \multicolumn{4}{|l|}{ Previous infection $\S$} \\
\hline No & $346 / 584(59.2)$ & $315 / 459(68.6)$ & $31 / 125(24.8)$ \\
\hline Yes & $50 / 145(34.5)$ & $46 / 105(43.8)$ & $4 / 40(10.0)$ \\
\hline \multicolumn{4}{|l|}{ Primary COVID-19 vaccination" } \\
\hline No & 98/167 (58.7) & $88 / 123(71.5)$ & $10 / 44(22.7)$ \\
\hline Yes & $285 / 541(52.7)$ & $264 / 427(61.8)$ & $21 / 114(18.4)$ \\
\hline \multicolumn{4}{|c|}{ Primary COVID-19 vaccination or previous infection ${ }^{q, \S}$} \\
\hline Unvaccinated, no previous infection & $82 / 131(62.6)$ & $72 / 96(75.0)$ & $10 / 35(28.6)$ \\
\hline Unvaccinated, previous infection & $16 / 36(44.4)$ & $16 / 27(59.3)$ & $0 / 9(-)$ \\
\hline Vaccinated, no previous infection & $251 / 433(58.0)$ & $234 / 349(67.0)$ & $17 / 84(20.2)$ \\
\hline Vaccinated, previous infection & $34 / 108(31.5)$ & $30 / 78(38.5)$ & $4 / 30(13.3)$ \\
\hline \multicolumn{4}{|l|}{ Days since onset or test** } \\
\hline 5 & $160 / 237(67.5)$ & $142 / 179(79.3)$ & $18 / 58(31.0)$ \\
\hline 6 & $91 / 166(54.8)$ & $80 / 121(66.1)$ & $11 / 45(24.4)$ \\
\hline 7 & $75 / 144(52.1)$ & $74 / 111(66.7)$ & $1 / 33(3.0)$ \\
\hline 8 & $43 / 112(38.4)$ & $39 / 93$ (41.9) & $4 / 19(21.1)$ \\
\hline 9 & $27 / 70(38.6)$ & $26 / 60(43.3)$ & $1 / 10(10.0)$ \\
\hline $5-9$ & $396 / 729(54.3)$ & $361 / 564(64.0)$ & $35 / 165(21.2)$ \\
\hline
\end{tabular}

Abbreviation: NAAT = nucleic acid amplification test.

* Abbott BinaxNOW COVID-19 Ag (BinaxNOW) rapid antigen test.

† SARS-CoV-2 infection diagnosed by NAAT or antigen test. persons were classified as symptomatic if symptoms were reported during routine case interview or isolation follow-up call.

$\S$ Previous infection is defined as previous positive SARS-CoV-2 NAAT or antigen test result $>90$ days before current episode, irrespective of vaccination status. Among those who were vaccinated and with previous infection, 96 had an infection before completion of the vaccination series.

" Vaccinated was defined as being $\geq 14$ days after 2 doses of an mRNA COVID-19 vaccine (Pfizer-BioNTech or Moderna) or 1 dose of the Janssen (Johnson \& Johnson) COVID-19 vaccine. Compared with no vaccination; 21 persons with partial vaccination were excluded. Among vaccinated persons, 518 had completed a 2-dose COVID-19 mRNA vaccination series, and 23 had received 1 dose of the Janssen vaccine. Two hundred fifteen persons were $\geq 7$ days after a booster dose, among whom 127 of $215(59.1 \%)$ had a positive antigen test result (126 of 212 [59.4\%] among those aged $\geq 18$ years); 158 of 326 (48.5\%) other vaccinated persons had a positive test result (119 of 232 [51.3\%] among those aged $\geq 18$ years).

** Defined as days since symptom onset if symptomatic, or days since the initial NAAT or antigen test if asymptomatic.

even if a person is infectious after an initial diagnosis, the risk for transmission to others depends on other important factors, including the duration and proximity of contact with others, and proper use of a well-fitting mask $(7,8)$. Following CDC recommendations, ${ }^{\mathbb{S}}$ YKHC advised continued mask use for 10 days after symptom onset even if a person met the criteria for ending isolation after 5 days. The high percentage of positive antigen test results during the 5-9 days after symptom onset

\footnotetext{
$\overline{\$ S ~ h t t p s: / / w w w . c d c . g o v / c o r o n a v i r u s / 2019-n c o v / y o u r-h e a l t h / q u a r a n t i n e-~}$ isolation.html (Accessed February 12, 2022).
}

reinforces the importance of correct and consistent mask use during this period.

The findings in this report are subject to at least six limitations. First, only one follow-up antigen test was included in the analysis for each person; data were cross-sectional rather than longitudinal. However, the multivariable model accounted for changes in reported characteristics over time. Second, the timing of acquisition of asymptomatic infections was unknown, limiting interpretation of changes in positivity over time in this group. Third, information is still accruing on the correlation between antigen tests and virus culture over time. Correlation 
FIGURE. Proportion of Abbott BinaxNOW COVID-19 Ag rapid antigen test results positive 5-9 days after symptom onset or after a positive initial test result* for SARS-CoV-2, by symptom status ${ }^{\dagger}(\mathrm{N}=729)$ - Yukon-Kuskokwim Delta region, Alaska, January-February 2022

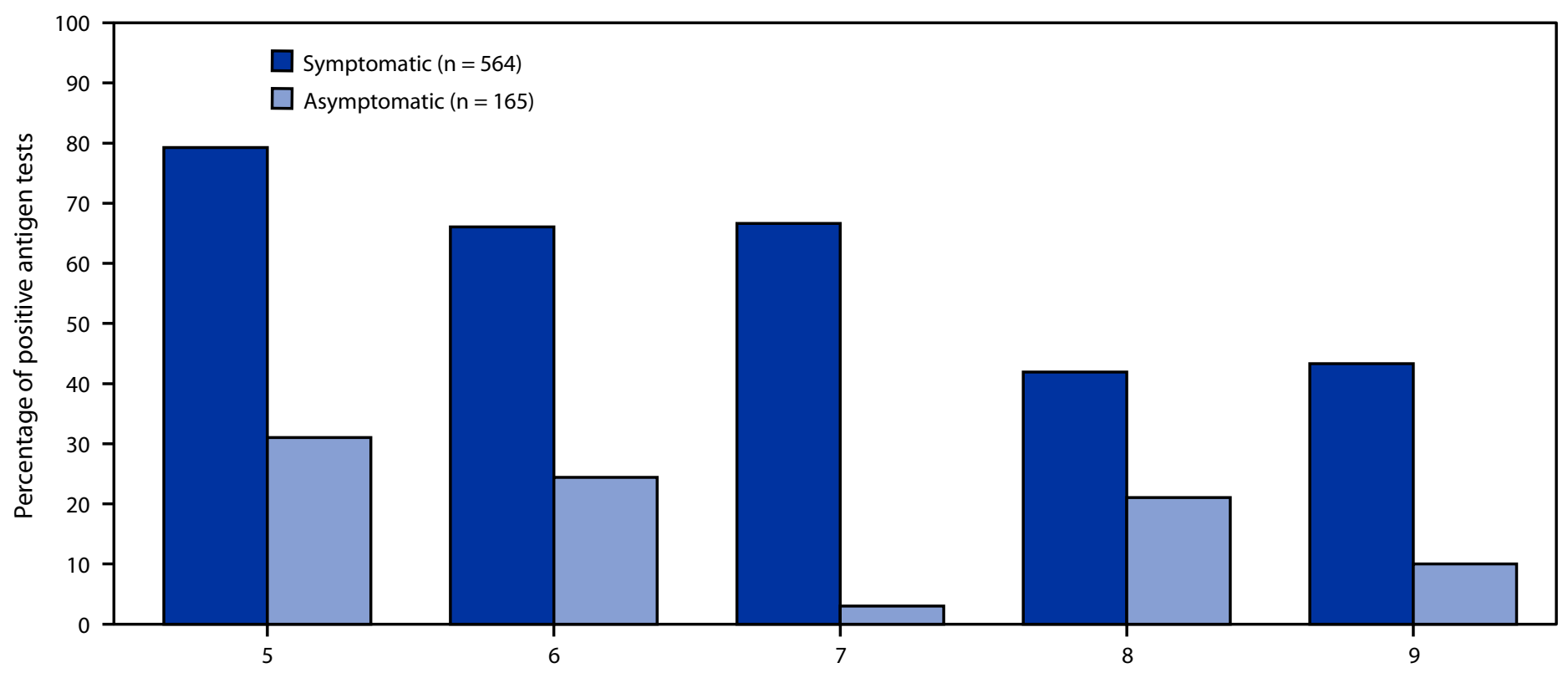

No. of days since symptom onset or initial positive test result (in asymptomatic patients)

\footnotetext{
* The initial test was a nucleic acid amplification test or antigen test for SARS-CoV-2. The chart summarizes the first follow-up antigen test result for each person during the 5-9 days after illness onset, or after the initial positive test result if asymptomatic.

† Persons are classified as symptomatic if symptoms were reported during routine interview or isolation follow-up call.
}

might vary between some tests or after introduction of the Omicron variant, although initial viral titers appear to be similar between Omicron and B.1.617.2 (Delta) variants. 99 Fourth, approximately $22 \%$ of persons who were 5-9 days after illness onset or an initial positive test result opted to have a follow-up antigen test, introducing potential selection bias. The sample was limited to persons whose symptoms were already resolving and might not be representative of all reported cases in other ways. Fifth, behavioral bias associated with the threshold for testing initially or during follow-up could possibly affect comparisons between individual characteristics. Finally, the results might be confounded by unmeasured factors.

Approximately $50 \%$ of antigen tests were positive 5-9 days after infection, but the percentage of positive results declined during this period and was lower after asymptomatic infection, after previous infection, and in persons who have completed a primary COVID-19 vaccine series. During the 10 days after infection, persons might be infectious to others and are recommended to wear a well-fitting mask when around others, and to avoid contact with those at elevated risk for severe disease, even if ending isolation after 5 days. Antigen tests might be a useful tool to guide recommendations for isolation after SARS-CoV-2 infection.

\section{Summary}

What is already known about this topic?

Positive rapid antigen test results after SARS-CoV-2 infection have been associated with the presence of viable virus, but the role of antigen tests in isolation guidance for persons with SARS-CoV-2 infection is unclear.

What is added by this report?

Between 5 and 9 days after symptom onset or after initial diagnosis with SARS-CoV-2 infection, $54 \%$ of persons had positive SARS-CoV-2 antigen test results. The proportion of positive results declined over time. Negative follow-up antigen test results were associated with asymptomatic infection, previous infection, and being vaccinated.

What are the implications for public health practice?

Antigen tests might be a useful tool to guide recommendations for isolation after SARS-CoV-2 infection.

\footnotetext{
99 https:/www.medrxiv.org/content/10.1101/2022.01.10.22269010v2
} 
TABLE 2. Associations between individual characteristics and a positive SARS-CoV-2 antigen test result 5-9 days after symptom onset or after a positive initial SARS-CoV-2 test result - Yukon-Kuskokwim Delta region, Alaska, January-February 2022

\begin{tabular}{|c|c|c|c|c|c|c|}
\hline \multirow[b]{3}{*}{ Characteristic } & \multicolumn{6}{|c|}{ Odds ratio $(95 \% \mathrm{Cl})$} \\
\hline & \multicolumn{2}{|c|}{$\begin{array}{l}\text { All infections* } \\
\qquad(\mathrm{N}=729)\end{array}$} & \multicolumn{2}{|c|}{$\begin{array}{l}\text { Symptomatic infections* } \\
\qquad(n=564)\end{array}$} & \multicolumn{2}{|c|}{$\begin{array}{l}\text { Asymptomatic infections* } \\
\qquad(n=165)\end{array}$} \\
\hline & Unadjusted & Adjusted $^{\dagger}$ & Unadjusted & Adjusted $^{\dagger}$ & Unadjusted & Adjusted $^{\dagger}$ \\
\hline Symptomatic infection $\$$ & $6.61(4.38-9.96)$ & $9.63(6.03-15.37)$ & - & - & - & - \\
\hline $\begin{array}{l}\text { Age group, yrs } \\
<18 \text { (Ref) } \\
18-49^{\natural} \\
\geq 50^{9}\end{array}$ & $\begin{array}{r}\text { Ref } \\
1.17(0.83-1.66) \\
1.55(1.00-2.42)\end{array}$ & $\begin{array}{r}\text { Ref } \\
1.2(0.78-1.85) \\
1.62(0.95-2.76)\end{array}$ & $\begin{array}{r}\text { Ref } \\
0.95(0.63-1.45) \\
1.32(0.78-2.24)\end{array}$ & $\begin{array}{r}\text { Ref } \\
1.05(0.65-1.71) \\
1.58(0.87-2.86)\end{array}$ & $\begin{array}{r}\text { Ref } \\
1.39(0.59-3.24) \\
1.13(0.34-3.72)\end{array}$ & $\begin{array}{r}\text { Ref } \\
1.93(0.69-5.45) \\
1.36(0.33-5.61)\end{array}$ \\
\hline \multicolumn{7}{|c|}{ Vaccination and previous infection status } \\
\hline $\begin{array}{l}\text { Previous infection }{ }^{* *} \\
\text { Primary COVID-19 } \\
\text { vaccination }^{\dagger \dagger}\end{array}$ & $\begin{array}{l}0.36(0.25-0.53) \\
0.78(0.55-1.11)\end{array}$ & $\begin{array}{l}0.30(0.19-0.46) \\
0.60(0.39-0.93)\end{array}$ & $\begin{array}{r}0.36(0.23-0.55) \\
0.64(0.42-1)\end{array}$ & $\begin{array}{r}0.29(0.18-0.46) \\
0.6(0.36-0.99)\end{array}$ & $\begin{array}{l}0.34(0.11-1.0) \\
0.77(0.33-1.8)\end{array}$ & $\begin{array}{l}0.37(0.11-1.19) \\
0.67(0.26-1.76)\end{array}$ \\
\hline $\begin{array}{l}\text { Unvaccinated, no } \\
\text { previous infection (Ref) }\end{array}$ & Ref & Ref & Ref & Ref & Ref & Ref \\
\hline $\begin{array}{l}\text { Primary COVID-19 } \\
\text { vaccination, no } \\
\text { previous infection }\end{array}$ & $0.82(0.55-1.23)$ & $0.60(0.37-0.99)$ & $0.68(0.41-1.13)$ & $0.68(0.39-1.19)$ & $0.63(0.26-1.57)$ & $0.5(0.18-1.42)$ \\
\hline $\begin{array}{l}\text { Primary COVID-19 } \\
\text { vaccination, previous } \\
\text { infection } \$ \S\end{array}$ & $0.28(0.16-0.47)$ & $0.17(0.09-0.33)$ & $0.21(0.11-0.4)$ & $0.16(0.08-0.34)$ & $0.39(0.11-1.39)$ & $0.30(0.07-1.29)$ \\
\hline \multicolumn{7}{|c|}{ No. of days after symptom onset until positive SARS-CoV-2 antigen test result } \\
\hline $5^{\text {ๆศ }}$ & $3.31(1.9-5.75)$ & 6.39 (3.39-12.03) & $5.02(2.68-9.38)$ & $6.84(3.49-13.43)$ & $4.05(0.48-34.41)$ & $4.11(0.45-37.37)$ \\
\hline 6า9 & $1.93(1.09-3.42)$ & $3.39(1.78-6.46)$ & $2.55(1.35-4.81)$ & $3.16(1.61-6.23)$ & $2.91(0.33-25.63)$ & $3.18(0.34-29.67)$ \\
\hline $7^{1199}$ & $1.73(0.97-3.1)$ & $2.85(1.48-5.47)$ & $2.62(1.37-4.99)$ & $3.47(1.74-6.93)$ & $0.28(0.02-4.95)$ & $0.34(0.02-6.29)$ \\
\hline 8था & $0.99(0.54-1.83)$ & $1.24(0.63-2.42)$ & $0.94(0.49-1.82)$ & $1.18(0.59-2.35)$ & $2.4(0.23-24.96)$ & $2.25(0.19-26.76)$ \\
\hline 9 (Ref) & Ref & Ref & Ref & Ref & Ref & Ref \\
\hline
\end{tabular}

Abbreviations: NAAT = nucleic acid amplification test; Ref = referent group.

* SARS-CoV-2 infection diagnosed by NAAT or antigen test. Persons were classified as symptomatic if symptoms were reported during routine case interview or isolation follow-up call.

${ }^{\dagger}$ Adjusted for age group, days since symptom onset or positive test result, previous infection status, previous vaccination status, and whether symptoms were reported.

$\S$ Compared with asymptomatic infection. Adjusted analyses excluded 21 persons (14 symptomatic and seven asymptomatic).

" Compared with children and adolescents (aged $<18$ years). Adjusted analyses excluded 21 persons (14 symptomatic and seven asymptomatic).

** Defined as previous positive SARS-CoV-2 NAAT or antigen test result $>90$ days before current episode, irrespective of vaccination status.

${ }^{++}$Vaccinated was defined as being $\geq 14$ days after 2 doses of an mRNA COVID-19 vaccine (Pfizer-BioNTech or Moderna) or 1 dose of the Janssen (Johnson \& Johnson) COVID-19 vaccine. Compared with no COVID-19 vaccination. Excluded 21 persons with partial vaccination (14 symptomatic infections and seven asymptomatic infections). Among 541 vaccinated persons, the adjusted odds ratio for a positive test result after being $\geq 7$ days after a booster dose compared with no booster was $1.69(95 \% \mathrm{Cl}=1.13-2.52)$, after adjusting for age group, days since symptom onset or positive test result, previous infection status, and whether symptoms were reported.

$\$ \$$ Compared with unvaccinated without previous infection. Excluded 21 persons with partial vaccination, and 36 unvaccinated persons with previous infection (overall 57 excluded, 41 symptomatic infections and 16 asymptomatic infections).

१ी Compared with day 9, where the day is defined as symptom onset (if symptomatic) or initial positive NAAT or antigen test result (if asymptomatic). Adjusted analyses excluded 21 persons (14 symptomatic and seven asymptomatic).

\section{Acknowledgments}

Jennifer Amigliore, Chelsey Beans-Polk, Faith Brink, Katherine Crow, Fisnike Dalipi, Martha Flores, Jackson Grimes, Joseph Klejka, Russell Lamont, Alyssa Leary, Helen Leinberger, Genevieve Maczynski, Kelly O’Brien, Jennifer Pak, Christine Richman, Franceska Robinson, Emily Tracy, Moses Tulim, Jeri Zapotosky, Yukon-Kuskokwim Health Corporation; Prabasaj Paul, Division of Healthcare Quality Promotion, National Centers for Emerging and Zoonotic Diseases, CDC; Eric Mooring, CDC COVID-19 Emergency Response Team; Joseph McLaughlin, Alaska Department of Health and Social Services.
Corresponding author: Ian D. Plumb, iplumb@cdc.gov.

${ }^{1}$ Yukon-Kuskokwim Health Corporation, Bethel, Alaska; ${ }^{2}$ Division of Preparedness and Emerging Infections, National Center for Emerging and Zoonotic Diseases, CDC; ${ }^{3}$ Division of Viral Diseases, National Center for Immunization and Respiratory Diseases, CDC; ${ }^{4} \mathrm{CDC} C O V I D-19$ Emergency Response Team.

All authors have completed and submitted the International Committee of Medical Journal Editors form for disclosure of potential conflicts of interest. No potential conflicts of interest were disclosed. 


\section{References}

1. Killingley B, Mann A, Kalinova M, et al. Safety, tolerability and viral kinetics during SARS-CoV-2 human challenge. In Review [Preprint posted online February 1, 2022]. https://www.researchsquare.com/article/ rs-1121993/v1

2. Buder F, Bauswein M, Magnus CL, et al. Contribution of high viral loads, detection of viral antigen and seroconversion to severe acute respiratory syndrome coronavirus 2 infectivity. J Infect Dis 2022;225:190-8. PMID:34427652 https://doi.org/10.1093/infdis/jiab415

3. Almendares O, Prince-Guerra JL, Nolen LD, et al.; CDC COVID-19 Surge Diagnostic Testing Laboratory. Performance characteristics of the Abbott BinaxNOW SARS-CoV-2 antigen test in comparison to real-time reverse transcriptase PCR and viral culture in community testing sites during November 2020. J Clin Microbiol 2022;60:e0174221. PMID:34705535 https://doi.org/10.1128/JCM.01742-21

4. Jones TC, Biele G, Mühlemann B, et al. Estimating infectiousness throughout SARS-CoV-2 infection course. Science 2021;373:eabi5273. PMID:34035154 https://doi.org/10.1126/science.abi5273
5. Routsias JG, Mavrouli M, Tsoplou P, Dioikitopoulou K, Tsakris A. Diagnostic performance of rapid antigen tests (RATs) for SARS-CoV-2 and their efficacy in monitoring the infectiousness of COVID-19 patients. Sci Rep 2021;11:22863. PMID:34819567 https://doi.org/10.1038/ s41598-021-02197-z

6. Korenkov M, Poopalasingam N, Madler M, et al. Evaluation of a rapid antigen test to detect SARS-CoV-2 infection and identify potentially infectious individuals. J Clin Microbiol 2021;59:e0089621. PMID:34213977 https://doi.org/10.1128/JCM.00896-21

7. Andrejko KL, Pry J, Myers JF, et al. Predictors of severe acute respiratory syndrome coronavirus 2 infection following high-risk exposure. Clin Infect Dis 2021. Epub December, 21, 2021. PMID:34932817 https:// doi.org/10.1093/cid/ciab1040

8. Andrejko KL, Pry JM, Myers JF, et al.; California COVID-19 CaseControl Study Team. Effectiveness of face mask or respirator use in indoor public settings for prevention of SARS-CoV-2 infection-California, February-December 2021. MMWR Morb Mortal Wkly Rep 2022;71:212-6. PMID:35143470 https://doi.org/10.15585/mmwr. $\mathrm{mm} 7106 \mathrm{e} 1$ 